\title{
Estrategias educativas de las clases medias en urbanizaciones cerradas del Gran Buenos Aires
}

Introducción ${ }^{1}$

En este artículo se analiza la relación entre los modelos de socialización y nuevos estilos residenciales (countries y barrios cerrados), a través de las estrategias de consolidación y ascenso social de sectores medios y

En el artículo se analizan las diferentes estrategias de ascenso y consolidación social de las nuevas clases medias. Específicamente las estrategias educativas de los actores a partir de la emergencia urbanizaciones cerradas. Si bien el fenómeno no es nuevo en Argentina, el boom inmobiliario

ha sido acompañando por una oferta educativa nueva en la zona. La primera estrategia se realiza a través de colegios tradicionales; la segunda, a través de la opción por una educación orientada a lo vincular; finalmente, la elección del "medio pelo" dentro de un modelo mercantil. Se finaliza con una reflexión sobre las posibilidades de una nueva integración entre sectores medios y medios altos a partir de estos nuevos espacios de socialización.

Palabras clave: estrategias educativas, sectores medios, segregación espacial, reproducción social, modelos de socialización.

- Investigadora docente de la Universidad Nacional de General Sarmiento y de la Universidad de Buenos Aires. Becaria CLACSOAsdi, concurso "Fragmentación social, crisis política e institucional" $200 \mathrm{I}$.

cdelcueto@hotmail.com medios-altos. En particular, las estrategias de los actores en relación con la educación de sus hijos y con los criterios de elección de las instituciones educativas. ¿Existen estrategias diferenciadas entre sectores consolidados y sectores en ascenso? ¿Cómo se realizan las elecciones de las instituciones escolares? ¿Qué criterios se utilizan en esta elección?

En las últimas dos décadas, la Argentina ha sufrido una serie de transformaciones profundas, tanto en su estructura económica y social como en la articulación entre Estado, mercado y sociedad. Estas

I. En este artículo se presentan algunos resultados del trabajo de investigación en el marco de mi tesis en la Maestría en Sociología de la Cultura y Análisis Cultural, IDAES, Universidad Nacional de General San Martín, bajo la dirección de la doctora Maristella Svampa.

Espiral, Estudios sobre Estado y Sociedad

Vol. XI No. 3I \Septiembre / Diciembre de 2004249 
transformaciones suponen, al mismo tiempo, cambios en los modos de interacción y los modelos de socialización entre los diferentes sectores sociales.

En relación con las clases medias, a partir de mediados de los años noventa, se produce una creciente polarización y un amplio distanciamiento entre "ganadores" y "perdedores" del nuevo modelo²(Svampa, 2001). La nueva estratificación conforma dos franjas: la primera, más reducida, corresponde a los "ganadores", grupo que estaría conformado por las élites planificadoras, los sectores gerenciales y profesionales. La otra franja, compuesta por los "perdedores", la integrarían, entre otros, importantes sectores de la clase media tradicional y de servicios que han experimentado trayectorias de empobrecimiento. En esta nueva dinámica, mientras parte de las clases medias se reduce por una movilidad social descendente que expulsa de este colectivo a importantes sectores; otros protagonizan trayectorias de ascenso social beneficiados por una mejor articulación con las nuevas estructuras del modelo. "El nuevo campo de fuerzas societal designa así menos una estructura ya consolidada, que un proceso atravesado por grandes tendencias, suerte de polos magnéticos que arrastra a diferentes grupos sociales hacia uno u otro costado, hacia la adquisición de posiciones ventajosas o hacia la pérdida de status; en el lenguaje de los actores, hacia la ‘salvación' o la “caída”' (Svampa, 2001: 26). Esta polarización al interior de las clases medias implica, al mismo tiempo, una polarización de estilos residenciales y modelos de socialización. Desde la perspectiva de los "ganadores", el proceso de suburbanización que comenzó a extenderse en Argentina a partir de 1989, que tiene por protagonistas centrales a parte de las clases medias

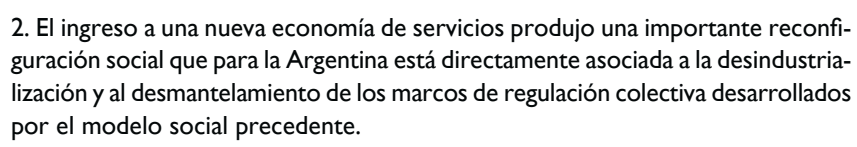

2. El ingreso a una nueva economía de servicios produjo una importante reconfiguración social que para la Argentina está directamente asociada a la desindustrialización y al desmantelamiento de los marcos de regulación colectiva desarrollados por el modelo social precedente. 
y altas, ilustra de manera privilegiada la nueva dinámica social. Asimismo, como consecuencia de la espacialización de las relaciones sociales, se conforman nuevos modelos de socialización caracterizados por la constitución de círculos homogéneos. De este modo, la proliferación de esta nueva forma de habitar muestra el pasaje de una matriz societal más rígida y jerárquica que aquella que caracterizara a la Argentina durante gran parte del siglo XX.

Si bien el fenómeno de las urbanizaciones cerradas es de larga data (Ballent, 1999), el boom inmobiliario que se produjo en la última década ha sido acompañando por una oferta educativa principalmente "bilingüe" que se estableció en las cercanías de ese tipo de desarrollos inmobiliarios, como correlato de un conjunto de nuevas aspiraciones sociales. De modo que se trata de un fenómeno novedoso y poco explorado.

Podemos hablar, entonces, de un nuevo escenario caracterizado por unas clases medias que optaron por residencias cercadas e internamente homogéneas y, al mismo tiempo, un sistema educativo que en su última reforma ya no tiene como uno de sus principales objetivos la promoción de estos sectores (Tiramonti, Suasnabar, 2000). De ello resulta un sistema que refuerza la fragmentación de los servicios educativos diagnosticada en los años ochenta (Braslavsky, 1985); un sistema con circuitos educativos diferenciados, que impide el contacto de los diferentes sectores sociales.

En la primera parte de este artículo se presenta una caracterización de la oferta educativa destinada a estos sectores, así como un agrupamiento de los colegios con sus principales características. En la segunda y tercera parte se analizan las diferentes estrategias educativas de las nuevas clases medias a partir de entrevistas realizadas en el Partido de Pilar, ubicado en la zona norte del conurbano bonaerense, geografía en donde los desarrollos inmobiliarios de urbanizaciones cerradas han tenido mayor crecimiento. 
Por último, la cuarta parte está concentrada en las implicancias y alcances de las diferentes estrategias educativas en el contexto de un fenómeno novedoso como es el de las urbanizaciones privadas.

La estrategia metodológica utilizada fue predominantemente cualitativa. En primer lugar, se utilizaron datos primarios a través de entrevistas semi estructuradas a madres con hijos en edad escolar residentes en countries y barrios cerrados. Se realizaron un total de 12 entrevistas, distribuidas de la siguiente manera: cuatro entrevistas correspondientes a familias que enviaban a sus hijos a colegios de tipo tradicional; cinco entrevistas a familias que enviaban a sus hijos a colegios de tipo vincular y tres entrevistas a familias cuyos hijos asistían a colegios con un perfil mercantil.

Debido a que los casos por analizar se seleccionaron a partir de un proceso de muestreo teórico, se tuvieron en cuenta el tipo de urbanización de residencia y el tipo de institución elegida como criterios para la conformación de los grupos que se compararían.

En relación con el primer criterio, es necesario tener en cuenta que las urbanizaciones pueden ser clasificadas de acuerdo con los segmentos de las clases medias a los que están destinados (Svampa, 2001). Básicamente, se ha trabajado con tres tipos de urbanizaciones cerradas: countries antiguos (décadas de 1930 y 1970); countries recientes (década de 1990) y barrios privados. Los countries antiguos o clubes de campo se iniciaron en la década de 1930 y cobraron nuevo impulso en los años setenta. Concebidos originalmente como residencia de fin de semana, se caracterizan por una intensa vida social. Los habitantes de countries recientes se ubican principalmente dentro de sectores altos y medios-altos. En este caso, la mayoría de las familias optan por este estilo de vida como residencia permanente. Tanto los countries antiguos como los recientes cuentan con una 
importante infraestructura deportiva que incluye canchas de tenis, futbol, polo y golf. Los barrios privados constituyen el tercer tipo de oferta residencial que, al mismo tiempo, es la más difundida. A diferencia de los primeros, el mérito principal lo constituye la seguridad ya que en muy pocos casos sus residentes disponen de espacios de uso común. ${ }^{3}$

Con respecto al segundo criterio, resultó necesario caracterizar la diversidad de colegios que tenían como principales destinatarios a residentes en barrios privados y countries. Dado que la oferta educativa es heterogénea, fue necesario establecer diferentes criterios para la clasificación de instituciones. ${ }^{4}$ Con este objetivo se realizaron entrevistas no estructuradas a informantes calificados: especialistas en educación (3 entrevistas), directivos y docentes de los colegios instalados recientemente en la zona (6 entrevistas). Las mismas permitieron definir con mayor precisión los criterios para distinguir la oferta existente a partir de los discursos que sostienen las instituciones hacia los padres y potenciales familias.

Finalmente, se analizaron diferentes fuentes documentales entre las que se pueden mencionar los siguientes materiales secundarios: publicaciones sobre colegios priva-

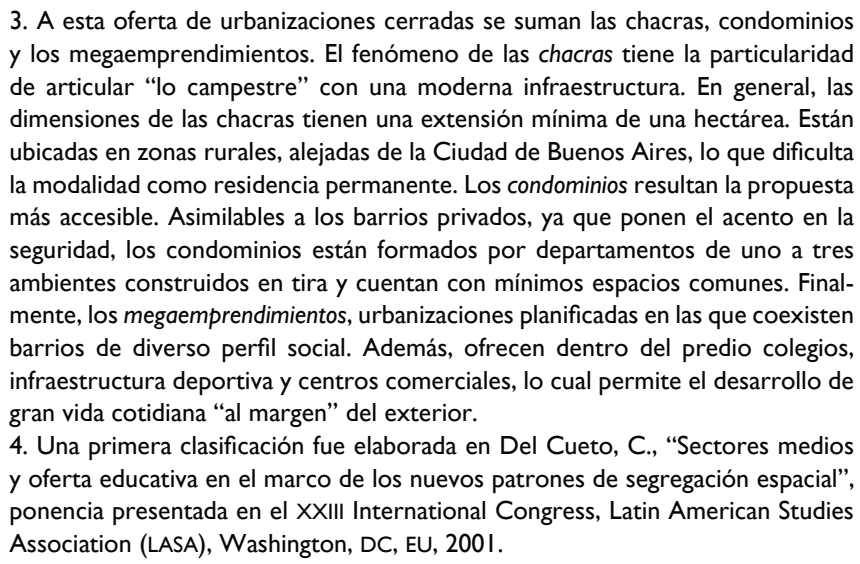


dos del Gran Buenos Aires, suplementos "Countries" de los periódicos Clarín y La Nación, páginas de Internet de los colegios de la zona. A partir del análisis de estas fuentes, se identificaron las diferentes propuestas educativas.

El trabajo de campo fue realizado entre marzo y agosto de 2002. El mismo se extendió más de lo previsto debido a lo que se llamó "pánico en los countries", vinculado a la posibilidad de saqueos en urbanizaciones cerradas. Sin embargo, cuando en las entrevistas se trató el tema del "miedo a la invasión", se respondía sistemáticamente que eso se había exagerado desde los medios de comunicación. Por otra parte, el crecimiento de los secuestros extorsivos generaba inquietud entre los entrevistados. Con el tiempo, fue posible volver a contactar a residentes dispuestos a las entrevistas. Nuevamente surgieron dificultades para concertar entrevistas cuando la zona de Pilar sufrió inundaciones por las fuertes lluvias ocurridas durante la segunda semana de abril, de modo que se reinició el proceso de entrevistas a comienzos de mayo.

\section{Características de la oferta educativa con "entorno country"}

Dentro de la oferta educativa con "entorno country" se pueden distinguir dos modalidades de instalación: una consiste en establecimientos que se encuentran en las cercanías de urbanizaciones privadas y otra de colegios ubicados dentro de countries. ${ }^{5}$

5. A estos últimos asisten 2,000 alumnos, de los cuales la mitad corresponde a residentes de la urbanización en la que están instalados (Clarín 8/4/00). Actualmente funcionan 10 colegios dentro de clubes de campo y se proyecta la construcción de 7 más. Según estimaciones de la Dirección de Educación de Gestión Privada del gobierno de la Provincia de Buenos Aires, de los 108 colegios privados ubicados en las zonas norte y oeste del conurbano bonaerense, $4 I$ abrieron sus puertas en los últimos 5 años en las cercanías de urbanizaciones cerradas (La Nación 15/04/0I). La zona con más oferta de este tipo es el Partido de Pilar que ha sido escenario de manera emblemática del crecimiento de countries y barrios cerrados. 
Si bien en un primer acercamiento a esta oferta nos parece estar frente a un panorama uniforme, una vez que nos acercamos a las particularidades de los servicios educativos nos encontramos con un paisaje diferente. A partir de entrevistas a padres y docentes hemos podido establecer ciertas distinciones dentro de la oferta teniendo en cuenta si el proyecto es laico o laico con orientación religiosa; si se privilegia el eje vincular o el académico. Se entiende por vincular cuando se presta atención a las relaciones entre los estudiantes: generalmente se apunta a la formación en los valores, a "formar buenas personas", o a que el niño "esté feliz en la escuela". Las instituciones cuyo eje es lo académico, en cambio, enfatizan principalmente la excelencia y el alto rendimiento académico. En la mayoría de los casos, por tratarse de segundas sedes, se ha tomado en cuenta la fecha de fundación de la sede original. Por último, el valor de la cuota.

A continuación, se presentan las principales características de cada grupo de colegios.

\section{Colegios tradicionales y el modelo de la excelencia}

El modelo de la excelencia incluye colegios con una fuerte trayectoria en el área educativa, con prestigio y "nombre propio", y con una antigüedad de más de cincuenta años. Se trata de instituciones que no poseen necesariamente una política de promoción para conseguir ampliar la matrícula sino que más bien la matrícula se acerca a ellos. A este tipo de colegios concurren en su mayoría sectores altos y mediosaltos. El aspecto tradicional está ligado, sobre todo, a que son colegios a los que han asistido padres y abuelos, primos y tíos de los estudiantes que concurren en la actualidad. De alguna manera, ser alumno de la institución forma parte de la tradición familiar. La composición de la matrícula no

En este partido la matrícula en colegios en predios cercados o cercanos a ellos asciende a 4,582 estudiantes. 
siempre está constituida por residentes de urbanizaciones cerradas sino que proviene de familias que viven en barrios "abiertos" de zona norte del conurbano bonaerense. Cabe aclarar que considerar a estas instituciones como "tradicionales" no supone necesariamente criterios tradicionales en términos pedagógicos. Dentro de este eje, se actualizan permanentemente e incorporan nuevas técnicas en el proceso de enseñanza-aprendizaje. Son colegios cuya cuota promedio es de 600 pesos $^{6}$ para la Educación General Básica $\left(\mathrm{EGB}^{7} \mathrm{y}\right.$ asciende más de 1,000 para el Polimodal. Incluyen en sus currículos viajes al exterior, a través de competencias deportivas, actividades de intercambio cultural, o para la realización de cursos de perfeccionamiento en idiomas. Dentro de este grupo podemos encontrar tanto colegios laicos como laicos con orientación religiosa. Sin embargo, como en su mayoría se trata de instituciones cuyo eje es lo académico, lo religioso suele quedar relegado. En este sentido, aparece un fuerte discurso que destaca la "excelencia" del proyecto y las posibilidades que brindan sus Bachilleratos Internacionales, ${ }^{8} \mathrm{de}$ ingreso a universidades privadas del exterior y del país.

\footnotetext{
6. Cabe aclarar que durante el modelo de convertibilidad un peso equivalía a un dólar. En la actualidad la moneda estadounidense se cotiza aproximadamente en tres pesos.

7. La última reforma educativa en Argentina se instituye legalmente con la Ley Federal de Educación en el año 1993. La misma apuntó a la descentralización definitiva del sistema que transfiere a las jurisdicciones provinciales la responsabilidad por la gestión, administración y financiamiento de las escuelas y las instituciones superiores no universitarias. Se crea la Educación General Básica (EGB) dividida en tres ciclos de tres años de duración, el último de los cuales corresponde a los dos primeros años de la anterior educación secundaria. En la antigua estructura el nivel primario tenía una duración de siete años y el secundario cinco. De este modo, se extiende la obligatoriedad a 10 años (un año de preescolar y nueve de educación básica). Los últimos tres años de la antigua educación media se transforman en un ciclo Polimodal no obligatorio con cinco orientaciones.

8. Se trata de colegios que siguen el plan de estudios aceptado por escuelas europeas y norteamericanas. Son de doble jornada y en los últimos dos años se prepara en el turno vespertino a los estudiantes para un examen que se evalúa fuera del país. El sistema de calificación incluye puntos extra por desempeño y por elaboración de ensayos en disciplinas a elección. Se otorga un título internacional que permite el ingreso a universidades del exterior.
} 
Estas propuestas enfatizan el desarrollo individual de la persona. Los valores que se sostienen son el rendimiento, la excelencia en lo académico, en lo deportivo, en lo artístico e incluso en el servicio comunitario. En efecto, al mismo tiempo que se refuerza el individualismo, de manera complementaria se llevan adelante actividades relacionadas con el trabajo solidario. Como parte del plan curricular y en el marco del Bachillerato Internacional, se llevan adelante actividades incluidas en el programa CAS (Creatividad, Acción y Servicio) a través del cual los estudiantes participan en diferentes programas de ayuda desarrollando proyectos propios en hospitales, hogares de ancianos, parroquias, escuelas estatales y hogares de niños, entre otros.

En cuanto a lo disciplinario, se implementa la autodisciplina como mecanismo para mantener la buena conducta en la institución. Así, se trata de la internalización de normas acompañada con una fuerte presencia de los adultos a partir del aprendizaje del autocontrol. Este sistema se orienta principalmente a la auto-responsabilidad, de modo tal que los jóvenes aprenden a ser ellos mismos su propia autoridad.

\section{Colegios recientes y el modelo vincular}

Dentro de este grupo encontramos colegios que tienen una trayectoria de más de 20 años. Ofrecen formación laica o laica con orientación religiosa. En el caso de esta última los niños tienen catequesis como una materia más del currículo, pero es opcional. Reciben tanto a practicantes católicos como a no católicos, y se ubican en un término medio entre la oferta religiosa y la bilingüe.

$\mathrm{Su}$ rasgo distintivo es el acento en el eje vincular. Si bien se destaca la formación académica, se enfatiza la educación "para la vida", y sobre todo "formar buenas personas". En este sentido, se otorga importancia al clima de compañerismo, más que a la competencia, y el disfrute en 
el desarrollo de las actividades escolares. El énfasis en lo vincular a veces lleva a implementar técnicas pedagógicas menos actualizadas, que se manifiestan en la disposición de los estudiantes dentro del aula y en algunas modalidades para el dictado de algunas materias. El fundamento de este perfil se sostiene en que, sin contención afectiva, el niño no es permeable al conocimiento. A diferencia del primer grupo de instituciones, cuentan con gabinete psicopedagógico que realiza acompañamiento en actividades concentradas en el trabajo sobre lo vincular. La mayoría de los colegios tiene una cuota promedio de $\$ 500$ mensual y se mantiene más o menos estable en todos los niveles. Otro rasgo que distingue a este tipo de instituciones es el vínculo que establecen con las familias. Tienden a fomentar la inclusión de los padres en la vida escolar, a que participen activamente de las actividades que propone el colegio y, del mismo modo, a incluir las iniciativas impulsadas desde las familias. Las madres entrevistadas también destacaron este rasgo del colegio, sus "puertas abiertas" para recibir sugerencias de los padres.

\section{Colegios recientes y el modelo mercantil}

Los colegios recientes que identificamos con el modelo mercantil tienen algo más de diez años de antigüedad. Uno de ellos posee sede sólo en Pilar y el otro tiene su sede original en la Ciudad de Buenos Aires y en la provincia de Mendoza. Constituyen una oferta de menor calidad. Ello se expresa no sólo en la presentación de los colegios sino también en la manera en que lo expresan los padres. Su principal virtud consiste en que ofrecen una cuota "accesible", de alrededor de $\$ 200$, e incluso realizan promociones para alumnos "fundadores" (media beca a lo largo de la vida escolar) con el fin de atraer matrícula. En general, se trata de colegios laicos. No ofrecen la opción de Bachillerato Internacional, pero están aceptados como sedes 
para rendir exámenes internacionales de inglés. El colegio que se inició en la Ciudad de Buenos Aires tuvo desde sus orígenes la imagen de "colegio para niños superdotados". Si bien en sus folletos aparecen fotos de prestigiosas universidades del exterior, no se explicita qué tipo de vínculo está vigente entre el colegio y tales universidades. A partir de su instalación en la zona de Pilar, el argumento fuerte de promoción fue brindar una "formación de excelencia" económicamente mucho más accesible que la del resto de las instituciones. A diferencia de los otros tipos de institución, en uno de ellos aparece de manera muy detallada las actividades y los contenidos de cada nivel. Por ejemplo, en nivel inicial figuran listados de palabras en inglés que los niños aprenderán durante el ciclo lectivo. En suma, esta opción es atractiva sobre todo para aquellas familias que disponen de menores recursos económicos y el éxito de esta propuesta se expresa en el vertiginoso crecimiento de la matrícula desde el momento de su instalación.

2. Clases medias y la apuesta por la educación como herramienta de consolidación

Las clases medias altas, que desarrollaron fuertes trayectorias de ascenso, y que son portadoras de capital cultural, realizan importantes apuestas en la formación de sus hijos. Nos encontramos, sin embargo, con diferentes estrategias de consolidación. Por un lado, las familias que han elegido para sus hijos colegios de tipo tradicional, donde se destaca una identificación fuerte con el proyecto del colegio. Están, por otra parte, quienes en principio también optaron por colegios tradicionales, pero que con el tiempo encontraron dificultades en el desempeño de sus hijos y decidieron cambiar de institución. En estos casos se observa una fuerte crítica al proyecto en cuanto a su exclusiva orientación académica. Al mismo tiempo, se destaca una valoración 
de otro tipo de elementos que no tuvieron en cuenta en el momento de la primera elección. Por su propia experiencia, han pasado de la identificación al distanciamiento y, finalmente, decidieron cambiar de colegio a sus hijos.

La identificación y el modelo de la excelencia

Respecto del primer grupo, nos referiremos a las familias que eran residentes de un country reciente. Dentro del predio funciona un colegio de tipo tradicional desde hace 12 años. Su matrícula está compuesta tanto por residentes del country como por estudiantes provenientes de zona norte de la Provincia de Buenos Aires y de la Ciudad de Buenos Aires. En las entrevistas no ha aparecido una descripción pormenorizada de otros colegios, lo que supondría que antes de la elección no se contó con información detallada sobre la oferta existente. Tampoco se relata el proceso de selección a partir de visitas a diferentes instituciones educativas de la zona. En los casos entrevistados este proceso está ligado a la comodidad de disponer de un "buen colegio" dentro del country, ya que para los padres la elección del colegio vino unida a la elección del lugar de residencia. Sin embargo, uno de los factores que ayudó en la decisión de comprar un lote en el country fue que hubiera un colegio prestigioso dentro del predio. De modo que en estos casos la opción de un nuevo estilo de vida incluía la institución escolar. No hubo sondeos por otros colegios en la zona sino que era el proyecto "cerrado" lo que resolvía cualquier posible disyuntiva. Entre las ventajas mencionadas figuran la "seguridad" y la "comodidad" que implica que los niños circulen de manera independiente dentro del country para asistir a clases. Todas las entrevistadas residentes del country mencionaron que una de las ventajas consistía en que sus hijos podían "ir y volver del colegio en bicicleta". De esta manera, se generaría en los niños el desarrollo de 
un sentimiento de autonomía al ser los mismos niños responsables de su propio tiempo.

Sin embargo, aun cuando la instalación del colegio dentro del country figura como una de las razones más importantes para la elección, las entrevistadas logran describir claramente los criterios que ayudaron a decidirse y en ese caso no siempre se vincula con la comodidad. Por otra parte, también se refieren con bastante fluidez a las actividades que realizan sus hijos en el colegio.

La identificación de los padres con el proyecto de la institución marca en cierta forma la fuerza que tiene este tipo de apuesta. Se trata de brindar a los hijos las mejores herramientas para el futuro. Dos de las entrevistadas, que desarrollaron fuertes trayectorias ascendentes y cursaron sus estudios en el circuito estatal, manifestaron en forma muy contundente su satisfacción con la elección realizada. En este grupo no aparecen críticas a la institución y la identificación con la propuesta es total.

Eo: ${ }^{9}$ No, educativa personalmente no, no tengo críticas, no, no. Me gusta... creo que un poco van las críticas en base a cómo van rindiendo los chicos, ¿no? O sea, vos ves que tus chicos rinden bien y todo está fenómeno, el chico presenta dificultades y empezás a ver como que si el colegio te responde, no te responde, yo no he tenido problemas, personalmente no he tenido problemas. Mi hijo mayor responde, el menor... está bueno iniciándose y pinta como que está queriendo responder, pero no, no tengo mayores críticas del colegio. Veo que es un colegio que está muy preocupado siempre en el sistema educativo, en como llevan a los chicos. Si hay alguna inquietud y uno se acerca... la respuesta es inmediata, al menos las veces que yo he tenido que recurrir, la respuesta fue inmediata. (Residente de un country reciente.) 
Con frecuencia se plantea un modelo de estímulo/respuesta: el niño "funciona" o "no funciona". En la medida en que los niños "responden" al ritmo institucional, y no se presentan dificultades de adaptación, no hay críticas al colegio.

En palabras de una de las informantes clave, son familias que "tienen al colegio como objeto de deseo", quieren que sus hijos asistan a ese colegio por su prestigio ya que les sirve como instrumento de consolidación y esto se expresa más claramente en referencia a las posibilidades que brinda el bachillerato internacional.

Eo: [...] Está bien, yo apuesto a todo eso, pero después cuando él consiga trabajo, hay que ver qué remuneración tiene en ese principio de trabajo, como para bancarse y seguir... es decir, siempre el principio es duro... eso lo veo porque fueron años de sacrificio y de laburo del padre. Pero qué sé qué suerte... sí, yo estoy apostando a una excelente educación, mi padre ha tenido una excelente educación, era un as en lo suyo y sin embargo la suerte no fue como la que tuvo mi marido, ¿entendés? Hay muchos profesionales que están haciendo tareas que no tienen nada que ver con lo suyo... porque están viviendo. Es decir, no es que yo sea pesimista, trato de ser realista, la realidad me muestra que hay casos que no son color de rosa. (Residente de un country reciente.)

La mayoría de estas elecciones se manifiestan en términos de apuesta ya que nada garantiza el futuro. Cabe señalar que gran parte de las entrevistas fueron realizadas durante 2002, de modo que el discurso en torno de la crisis y la inestabilidad estuvo muy presente en la mayoría de los entrevistados. Sin embargo, aun en el contexto de la crisis, lo último a lo que están dispuestos a renunciar es a la formación de sus hijos.

El distanciamiento y la opción por lo vincular

El segundo grupo está conformado por aquellos que eligieron colegios tradicionales, pero a partir de la experiencia 
escolar decidieron cambiar a sus hijos a instituciones que acentuaran el eje vincular. Entre las críticas más frecuentes se encuentra la desatención en la formación de valores relacionados con la contención afectiva.

Eo: Pero no así lo humano, yo siempre digo que para mí [...] es como una casa sin padres, que los padres se han ido de viaje. ¿Viste la casa donde los padres se han ido de viaje? [...] Porque... porque tienen... porque no hay contención humana, no hay contención afectiva. Si un chico viene y se divierte martirizando a otro ese otro no tiene a quién recurrir que haga efecto... efectivamente, valga la redundancia, para que se le ponga el límite a ese chico que también es sano y se refuerce el valor... por ejemplo, el chico le va a decir a la maestra y la maestra le dice "arreglate". (Residente de un country reciente.)

Críticas como la citada son realizadas con frecuencia por parte de familias que tienen cierta antigüedad de clase y que, por sus propios recorridos (escolarizados en instituciones de tipo tradicional), se permiten una mayor distancia con el proyecto educativo. Así, prefieren cambiar por colegios en donde haya menos acento en la competencia y menos presión en el rendimiento académico.

Antes de decidirse a cambiar de institución, los padres atravesaron experiencias de frustración en la vida escolar de sus hijos. No siempre la dificultad tiene que ver con seguir el ritmo de estudio que impone la institución sino que, a veces, tiene que ver con problemas de adaptación al grupo de compañeros. De alguna manera, los problemas de adaptación se perciben como dificultades del colegio para guiar y apoyar el desempeño de los alumnos.

Eo: Es mi eterna pelea, ¿no? Por eso cuando yo los saqué les dije "ojo, porque la limitación es de ustedes, no mi hijo, mi hijo sólo tiene dislexia, la limitación es de ustedes porque si ustedes se jactan de tener excelentes profesores". El profesor que es excelente es el que puede 
sacar adelante a cualquier chico, porque sacar adelante a los chicos que salen solos no es mérito del profesor, es mérito del chico, entonces... es mi eterna discusión [...] (Residente de un country reciente.)

Luego de este tipo de experiencias, las familias decidieron cambiar a los niños de colegio y renunciar a la formación de excelencia que proponen estas instituciones. El cambio implica no sólo una pérdida en términos de formación académica y artística sino también una ruptura con la trayectoria familiar. Recordemos que en varios casos los padres son ex alumnos de estas instituciones.

Eo: [...] Me parece que... yo no siento... yo no siento que haya perdido de ninguna de las dos maneras, para mí haberlos sacado del colegio que me costó muchísimo porque mi marido es ex alumno... ya tenía la camiseta puesta, para mí dije "bueno, la verdad que durante todos estos años tuvieron una oportunidad de esto y esto y no tuvieron la oportunidad de por ahí de ser educados más desde los valores como fui educada yo". (Residente de un country reciente.)

Como señalamos, en este distanciamiento hacia la institución por su exclusividad en el eje académico los padres se orientan hacia propuestas educativas que enfatizan el eje vincular.

Eo: $Y$ tratan de contemplar las situaciones que vive cada chico en particular y realmente vos te das cuenta que los conocen porque vos vas y decís alguna cosa y ellos dicen "pero, bueno, él tiene este tipo de actitudes o comportamientos". Y vos te das cuenta que lo conocen, no es que te están inventando y te están hablando de otro chico. Eh... eso es lo que más me atrae del colegio... (Residente de un barrio privado.)

La elección se basa, por un lado, en subsanar la falta de atención a la formación en "valores humanos", pero también se busca un estilo más "democrático". Como señalan Dubet 
y Martuccelli (1998), se trata de un modelo de socialización "contractual", menos opresivo, que gira alrededor de la motivación. Por otro lado, los padres sienten que tienen un lugar en la institución, que pueden acercarse y plantear libremente sus inquietudes. Si bien los colegios tradicionales disponen de mecanismos de inclusión de los padres en la vida de la comunidad educativa, en la mayoría de los casos se establece a partir de comisiones de padres y a través de canales más institucionalizados.

Los colegios elegidos dentro del modelo vincular también ofrecen Bachillerato Internacional que, en términos de perspectivas futuras, implica la posibilidad de continuar estudios universitarios en el exterior. Sin embargo, en ninguno de estos casos se presenta como alternativa efectiva. Igual que los primeros, prefieren pensar en términos de "dejar las puertas abiertas". Pero a diferencia de aquellos que envían a sus hijos a colegios tradicionales, la posibilidad es más lejana, realizan una evaluación más "realista" sobre la capacidad económica de sostener un proyecto de este tipo en el futuro. En este sentido, se valora la oferta universitaria nacional tanto privada como estatal.

\section{El "medio pelo" ${ }^{10}$ y el modelo mercantil}

En su mayoría, las familias que despliegan estrategias dentro de este modelo son residentes de barrios privados y countries antiguos. ¿Cuáles son las apuestas dentro de este grupo? En principio, señalemos que cuando se refieren a la elección del colegio mencionan, en primer lugar, el valor de la cuota. Esto no aparece de manera tan evidente en los

10. Recuperamos aquí la noción de "medio pelo" utilizado por Jauretche. Dicho sector estaba compuesto por clases altas venidas a menos y sectores medios en proceso de rápido ascenso social. En uno y otro caso se trata de imitar pautas de comportamiento propias de las clases altas. En este sentido, el "medio pelo" estaría conformado por aquellos sectores que imitando estas pautas viven en "la apariencia de una apariencia" (Jauretche, 1967: 309). 
otros grupos, en donde el patrón de elección se refiere más a la calidad de la educación tanto en el eje académico como en el vincular.

Eo: [...] La elección se hizo, dado que el colegio cuando empezó Constanza lanzó una promoción. Entonces había una promoción muy interesante que a las primeras $\mathbf{2 0 0}$ familias les hacían una media beca de por vida. Este... entonces dentro de todo cerraba con el proyecto de lo que uno espera de un colegio. Es decir, lo que ellos estaban planteando, digamos, cerraba en las necesidades y un poco lo... bueno, anotamos a Constanza pensando que era una prueba piloto durante jardín: "Si vemos que el colegio no nos termina de convencer en cuanto a sus aspectos morales y demás cuestiones siempre estamos a tiempo de sacarla". Todos los años hago una evaluación respecto de otros colegios a ver cómo va avanzando. Hay cosas que el colegio prometió y que después no cumplió.

Er: ${ }^{11}$ ¿Como cuáles?

Eo: Por ejemplo, una cosa que a mí me molestó mucho es el tema que dijeron "a las primeras 200 familias se les da la beca", este... al año siguiente eso siguió y siguió el mismo precio que nos estaba cobrando a nosotros durante 3 años más. Entonces, si vos lo anotastes (sic) al chico comprándole... o sea, dándole el apoyo porque la escuela estaba vendiendo el proyecto como una maqueta, todavía el colegio no estaba. Entonces a esas primeras 200 familias que él les dijo "yo prometo una media beca"... de alguna manera compensalo de otra forma contra aquellas que después vinieron dos años más tarde y están recibiendo el mismo beneficio. Yo en eso me siento estafada, isí? Entonces yo creo, si viene una persona "yo a los primeros 200 les doy tal premio" bien, da tal premio, si a los demás los querés beneficiar con otra cosa, están bien pero entonces a los primeros beneficialos con algo mayor que son los que te dieron el voto de confianza de entrada [...] Se mantuvo el precio de la cuota, que me parece muy lógico de acuerdo al problema económico que hay en el país, me parece lógico que no eleve la cuota 
demasiado. Eso me parece muy razonable y que ayude a que otros... de gente que por ahí no tiene la posibilidad de mandar a los chicos a un colegio carísimo pueda acceder a un buen colegio con una cuota razonable. Pero de ahí en más a los primeros que nosotros que fuimos los que le dimos el apoyo inicial, bueno "cobro una matrícula al 50 por ciento" por ejemplo, ¿sí? Somos los que nosotros le dimos el puntapié inicial, ¿verdad? Ayudamos a que arranque con el proyecto. (Residente de un barrio privado.)

Como señala Bourdieu (1998), los consumos ligados a la buena voluntad cultural se caracterizan por tomar la imitación —de las clases medias altas- por lo auténtico:

El consumo de la "imitación" es una especie de bluff inconsciente que engaña sobre todo al que lo practica, primer interesado en tomar la copia por el original y el relumbrón por lo auténtico, a la manera de los compradores de "imitaciones", de saldos o de ocasión, que quieren convencerse de que "es más barato y hace el mismo efecto" (Bourdieu, 1998: 327).

En este grupo aparece de manera más frecuente un periplo por diferentes instituciones que implica cálculos no sólo para afrontar el nivel de la cuota que se expresa en términos mercantiles, sino también se sopesa de manera minuciosa el equilibrio entre servicios ofrecidos y costos.

Eo. Vos generalmente no ves primero la lista de precios, vas a ver qué te ofrece el colegio: si tiene actividades extraprogramáticas, si tiene catequesis, si el colegio tiene un buen comedor... una de las cosas que me encantó del colegio W., por ejemplo, fue el comedor, lo organizado y la amplitud del comedor, acá todos los colegios pecan de poner lugares de comer demasiado estrechos, muy chiquitos, muy chiquitos. (Residente de un barrio privado.) 
Otras veces, la elección de este tipo de instituciones se presenta como la única posible debido a la inestabilidad laboral.

Eo: [...] No creo que haya colegios perfectos, en mi realidad y en mi situación económica también en el día de hoy es una de las pocas opciones que tengo en cuanto a lo económico, pero en cuanto a la calidad de educación que los chicos están recibiendo yo estoy muy contenta. (Residente de un country antiguo.)

La carrera universitaria de los hijos debería desarrollarse en el exterior, en instituciones privadas de Estados Unidos e Inglaterra.

Er: ¿Te imaginás a los chicos yendo a una universidad en el exterior? Lo que te gustaría, más allá de lo que elijan ellos...

Eo: Más que imaginármelo, lo veo como una cosa necesaria el día de mañana, yo creo que la educación acá es cada vez peor, tanto la universitaria que antes era excelente y vos escuchás que cada vez es peor.... (Residente de un barrio privado.)

Ellos esperan que sus hijos puedan continuar estudios superiores en universidades costosas, pero en el presente cuesta mantener un colegio cuya cuota es mucho más baja que la que tendrían que pagar en una universidad del exterior. $\mathrm{O}$ una vez elegida la universidad privada, dan por sentada la necesidad de realizar posgrados en el exterior. Aquí recuperamos a Jauretche cuando señala que el "medio pelo" tiende a reproducir pautas de conducta de sectores ubicados en la cima de la escala social, a través de la apariencia de una apariencia.

Vemos en este grupo que la elección sigue el patrón de la viabilidad económica, la cuota accesible, mientras que el costo de la educación no figura en la agenda de temas relevantes de otros grupos. De hecho, frente a problemas de 
orden económico las familias que apuestan a la consolidación prefieren reducir gastos en las actividades extracurriculares e incluso deciden cambiar de lugar de residencia, pero mantener el colegio de los hijos. Por otra parte, el modo en que describen las características de los colegios es impreciso: o bien se refieren a características edilicias, o bien describen la "excelencia" de la institución en términos indefinidos. En fin, parecen no tener herramientas para justificar su propia elección y, sin embargo, consideran que a partir de la educación que reciben sus hijos es posible acceder al éxito. Para Bourdieu (1998), el principio de la buena voluntad cultural es la distancia existente entre el conocimiento y el reconocimiento de la cultura. En este caso se consideran suficientes las características edilicias de colegios para dar cuenta de su calidad, y ésta es una diferencia sustantiva en relación con los grupos anteriores. En efecto, como señala Bourdieu: "La pequeña burguesía ascendente invierte su buena voluntad desarmada en las formas menores de las prácticas y de los bienes culturales legítimos [...] de la misma manera que despliega cantidades prodigiosas de energía y de ingenio para vivir, como vulgarmente se dice 'por encima de sus posibilidades"' (Bourdieu, 1998: 323). Es por ello que hemos definido esta tercera estrategia como la estrategia del "medio pelo" dentro de un modelo de educación mercantil.

\section{Comentarios finales}

En los últimos años, el sistema educativo argentino se ve afectado por la retracción progresiva del Estado en el diseño de las políticas sociales que, aunque de manera desigual, habían garantizado el bienestar de la población. Así, la insuficiente inversión estatal, el consiguiente deterioro de los salarios y las condiciones laborales de los docentes, sumados a la ausencia de políticas dirigidas a jerarquizar 
la formación de los maestros, derivan en una profunda declinación del sistema educativo. Estas tendencias se conjugan con las nuevas demandas a las que debe responder la escuela, no sólo cuantitativas, por el aumento de la población escolar, sino también cualitativas, por el espacio creciente que en las escuelas comienzan a ocupar las tareas asistencialistas como consecuencia de la pauperización del alumnado. Ante el deterioro de la calidad de la enseñanza en los establecimientos estatales, aquellos sectores de la población con mayor poder adquisitivo comienzan a volcarse a los establecimientos privados: la matrícula del sector pasa de $18.5 \%$ en 1955 a $30 \%$ a mediados de los años noventa (Morduchowicz, 1999). A su vez, y en forma simultánea a la expansión del sector privado, el sector público comienza a segmentarse en circuitos diferenciados que contrarrestan los efectos democratizadores del aumento de la matrícula reproduciendo la desigualdad social de los alumnos (Braslavsky, 1985). Las lógicas de mercado comienzan a invadir el sistema educativo en el espacio abierto por una deficiente intervención estatal, tanto en lo relativo a la equiparación de las condiciones de aprendizaje entre los establecimientos como al control de la interacción entre la oferta y la demanda educativa. La distribución de los alumnos entre escuelas públicas y privadas jerárquicamente diversas en cuanto al origen socioeconómico de los alumnos, el perfil de los docentes, la infraestructura, los modelos pedagógicos y la organización, depende estrictamente de las capacidades de elección de las familias, directamente ligadas a sus recursos económicos, culturales y sociales (Veleda, 2003; Filmus, 2001; Kessler, 2002).

Actualmente, las franjas "ganadoras" de las clases medias han sido protagonistas de un proceso de segregación espacial y sus elecciones educativas conforman nuevos patrones que responden y refuerzan la fragmentación ya existente en el sistema educativo. Al mismo tiempo, cada segmento 
se vuelve internamente más homogéneo. En este sentido, podemos recuperar la noción de Cohen (1998) de "apareamientos selectivos" es decir, que los lugares de socialización se constituyen en espacios en donde la asociación se realiza de manera selectiva entre "semejantes". Instituciones como la familia y la escuela tienden cada vez más a homogeneizarse, y las estrategias educativas desplegadas por las familias se vuelven más selectivas.

Si bien, en términos generales, no se cuestiona la capacitación e infraestructura de las instituciones destinadas a las franjas ganadoras de los sectores medios, se advierte la fragmentación que va sufriendo la educación, con brechas que se abren entre los que tienen más posibilidades y los más rezagados. Así, se pone en duda el papel de socialización, que lentamente van perdiendo la institución escolar y la experiencia educativa. De modo que esta problemática cobra nueva significación si se tiene en cuenta que en Argentina la escuela no sólo significó un instrumento privilegiado para el ascenso social sino que, al mismo tiempo, encarnó de manera emblemática aquel espacio en el cual era posible la integración de los más diversos sectores sociales.

Aquellos que apuestan a la consolidación social establecen relación con sectores medios-altos y altos, especialmente en los colegios tradicionales. En estos casos permanece explícitamente un discurso que valora la formación y se experimenta el gasto como una inversión para el futuro de sus hijos. Los sectores medios-altos en proceso de consolidación envían a sus hijos principalmente a colegios tradicionales y de hecho constituyen la mayor proporción de la matrícula. Parecería verificarse en esta elección una apuesta a la consagración recurriendo a colegios prestigiosos. Un segundo tipo de estrategia la despliegan aquellos que eligen instituciones por su acento en lo vincular; sin embargo, cabe aclarar que a veces esta elección se produce luego de intentar permanecer en colegios tradicionales. 
En los colegios de perfil vincular encontramos a sectores medios-altos que buscan una educación más contenedora afectivamente. En varios casos hemos registrado que en primer lugar han optado por instituciones tradicionales, pero que de acuerdo con su trayectoria se permiten un mayor distanciamiento con la institución y su prestigio.

Un tercer tipo de estrategia es el llevado adelante por franjas de las clases medias denominadas "medio pelo". Una clase media típica que no puede acceder, por el valor de las cuotas, a los colegios antes mencionados. En este sentido, si bien manejan información privilegiada para realizar sus elecciones educativas, su orientación seguiría el patrón de las apariencias. Dicha orientación parece ir en contra de la mayoría de los diagnósticos en los cuales se destaca la importancia del manejo de información en el acceso a circuitos educativos de calidad (Dubet, 1996). En efecto, aunque disponen de ventajas en relación con otros grupos sociales no son capaces de manejarlas a su favor. Apartadas de la educación estatal, al tener que gestionar individualmente la elección educativa, en algunos casos se ven más perjudicadas que beneficiadas y optan por una educación de menor calidad.

Si bien en la mayoría de los casos se percibe una fuerte apuesta al ascenso en la elección educativa - sobre todo cuando se eligen colegios tradicionales-, no se percibe lo mismo en lo relativo a la acumulación de capital social, es decir, en la ampliación de relaciones con otros padres provenientes de sectores más acomodados. $\mathrm{Al}$ menos por lo incipiente del proceso, la integración entre sectores sociales ubicados en diferentes posiciones sociales al nivel de las relaciones entre familias no siempre se establece en términos de vínculos fuertes. Lo que se produce en realidad es el afianzamiento de relaciones con "los semejantes". Incluso en los casos en donde hay una apuesta por el ascenso social y al establecimiento de relaciones con sectores altos, los adultos tienden a establecer relaciones entre semejantes. 
En donde sí se verifica un afianzamiento de las relaciones entre uno y otro sector es entre los niños. Como señala Mills: "Es en la 'generación siguiente' en las escuelas particulares donde se relajan y hasta se resuelven las tiranteces entre clases altas nuevas y viejas. Y por medio de esas escuelas, más que por cualquier otro recurso, las familias antiguas y las nuevas - cuando llega su tiempo- se convierten en una clase social consciente de sí misma" (Mills, 1993: 67). $\mathrm{Al}$ mismo tiempo, los colegios fomentan esta homogeneidad a través de un trabajo grupal sistemático que no siempre está ligado a una concepción grupal del aprendizaje sino más bien a ser parte de un grupo de homogéneo. Hay un interés en el desarrollo individual del estudiante, pero también hay interés en la conformación de un espíritu de cuerpo a través de estas actividades. De este modo, las estrategias educativas de las nuevas clases medias suponen no sólo la formación académica, es decir, la acumulación de capital cultural institucionalizado, sino también la acumulación de capital social para la generación siguiente. Dentro del fenómeno novedoso de los nuevos estilos residenciales, que supone al mismo tiempo nuevos modelos de socialización, el colegio privado se ha convertido en una extensión de la urbanización cerrada, y las formas de socialización anteriores fueron reemplazadas por la constitución de círculos sociales homogéneos. Sin embargo, las estrategias educativas en términos de consolidación y ascenso social no parecen ser muy diferentes de las que podemos encontrar en el resto de la sociedad. "Los grupos estamentalmente privilegiados no aceptan jamás sin reservas al parvenu — por semejante que sea su modo de vida al suyo-, sino únicamente a sus descendientes, los cuales han sido educados ya en las convenciones de clase" (Weber, 1996: 692). La integración entre sectores en ascenso y sectores medios-altos y altos sólo puede hacerse efectiva en el futuro y para la próxima generación. 
Bibliografía | Ballent, A., "La 'casa para todos': grandeza y miseria de la vivienda masiva", en Devoto, F. y M. Madero (directores), Historia de la vida privada en la Argentina, Buenos Aires, Taurus, 1999, t. III.

Braslavsky, C., La discriminación educativa en Argentina, Flacso-Grupo Editor Latinoamericano, Buenos Aires, 1985.

Braslavsky, C., "Transformaciones en curso en el sistema educativo argentino", en_Educación, equidad y competitividad económica en las Américas, Washington, Agencia Interamericana para la Cooperación y el Desarrollo, Organización de los Estados Americanos.

Bourdieu, P. y J-C Passeron, Los estudiantes y la cultura, Buenos Aires, Labor, 2000 [1973].

Bourdieu, P., "Le Capital social. Notes provisoires", en Actes de la Recherche en Sciences Sociales, París, núm. 3I, 1980.

__ La distinción. Criterios y bases sociales del gusto, Madrid, Taurus, 1988.

—- Capital cultural, escuela y espacio social, México, Siglo XXI Editores, 1998.

— y J-C Passeron, La reproducción. Elementos para una teoría del sistema de enseñanza, México, Fontamara, 1995.

__ "Las formas del capital. Capital económico, capital cultural y capital social", en Poder, derecho y clases sociales, Bilbao, Ed. Desclée, 2000.

Carli, S., "Clases medias, pedagogías psi y cultura escolar en la historia reciente de la Argentina", ponencia presentada en las Jornadas de Sociología, Facultad de Ciencias Sociales, Universidad de Buenos Aires, 2000.

Cohen, D., Riqueza del mundo, pobreza de las naciones, Buenos Aires, Fondo de Cultura Económica, 1998.

Del Cueto, C., "Sectores medios y oferta educativa en el marco de los nuevos patrones de segregación espacial", 
ponencia presentada en el XXIII International Congress, Bibliografía Latin American Studies Association (LASA), Washington, DC, EU, 200I.

Dubet, F., “L'exclusion scolaire: quelles solutions?" en Paugam, Serge (dir.), L'exclusion: l'état des savoirs, París, La Decouverte, 1996.

Dubet, F. y D. Martucelli, En la escuela. Sociología de la experiencia escolar, Buenos Aires, Losada, 1998.

Filmus, D., C. Kaplan, A. Miranda y M. Moragues, Cada vez más necesaria, cada vez más insuficiente, Buenos Aires, Santillana, 200I.

González Bombal, M. I. y M. Svampa, “Movilidad social ascendente $y$ descendente en las clases medias argentinas: un estudio comparativo", Buenos Aires, Siempro-Miño y Dávila, 2000 (en prensa).

Jauretche, A., El medio pelo en la sociedad argentina, Buenos Aires, A. Peña Lillo, 1967.

Kessler, Gabriel, La experiencia fragmentada. Estudiantes y profesores en las escuelas medias de Buenos Aires, Buenos Aires, IIPE-UNESCO, 2002.

Mills, C. W., La elite del poder, México, Fondo de Cultura Económica, 1993.

Morduchowicz, A., Estudio sobre la educación privada en la Argentina: historia, regulaciones y asignación de recursos públicos, Buenos Aires, Centro de Estudios para el desarrollo Institucional de la Fundación Gobierno y Sociedad, 1999 (mimeo).

Pinçon, M. y M. Pinçon-Charlot, Sociologie de la bourgeoisie, París, La Decouverte, 2000.

Tedesco, J. C., Educación y sociedad en la Argentina (I8801945), Buenos Aires, Solar, 1993.

Tiramonti, G. y C. Suasnabar, "La reforma educativa nacional en busca de una interpretación", en Revista Aportes para el Estado y la Administración Gubernamental, Buenos Aires, núm. 15, 2000. 
Bibliografía | Sebreli, J. J., Buenos Aires, vida cotidiana y alineación, Buenos Aires, Siglo XX, 1964.

Svampa, M., "Clases medias, cuestión social y nuevos marcos de sociabilidad", en Punto de Vista. Revista de Cultura, Buenos Aires, núm. 67, 2000.

—- Los que ganaron. La vida en los countries y barrios privados, Buenos Aires, Biblos, 2001.

_- "Las nuevas urbanizaciones privadas: sociabilidad y socialización", en varios autores, Sociedad y sociabilidad en la Argentina de los 90, Buenos Aires, Biblos, 2002.

Veleda, C., Debates y evidencias acerca de los mercados educativos Las familias y elección de la escuela en el conurbano bonaerense Mercados Educativos, Buenos Aires, CIPPEC, 2003.

Weber, Max, Economía y sociedad, México, Fondo de Cultura Económica, 1996. 